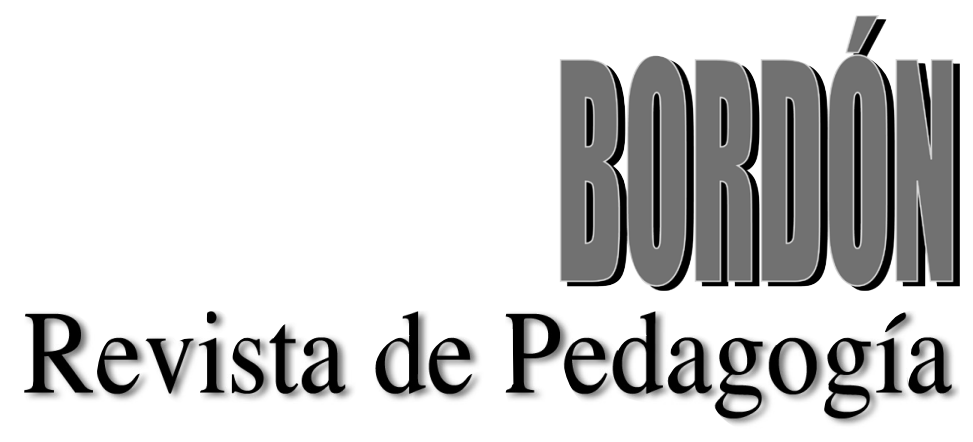

NÚMERO MONOGRÁFICO / SPECIAL ISSUE

Educación y arquitectura / Education and architecture

Teresa Romañá (editor invitado / guest editor)

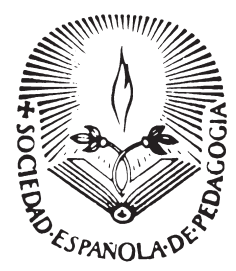

\author{
Volumen 68 \\ Número, 1 \\ 2016
}




\title{
DE LA EDUCACIÓn DEL ARQUITECTO A LA ARQUITECTURA DE LA EDUCACIÓn: Un DIÁLOGO IIIPRESCINDIBLE*
} From the education of the architect to the architecture of education: an essential dialogue

\author{
MAGDA SAURA CARULLA, JOSEP MUNTAÑOLA THORNBERG, \\ SERGI MÉNDEZ RODRÍGUEZY JÚLIA BELTRÁN BORRÀS \\ Universitat Politècnica de Catalunya
}

D01: 10.13042/Bordon.2016.68110

Fecha de recepción: 05/07/2015 - Fecha de aceptación: 14/10/2015

Autor de contacto / Corresponding Author: Josep Muntañola Thornberg. Email: jose.muntanola@upc.edu

INTRODUCCIÓN. El presente artículo pretende revisar las relaciones entre arquitectura y educación, no solamente desde una perspectiva que insista en la presencia de la arquitectura y el urbanismo en los currículos educativos contemporáneos, como es el caso de la tan imitada Finlandia, sino desde un punto de vista que insiste en que esta introducción modificaría toda la educación en su conjunto al incluir el espacio físico y social como dimensiones necesarias en las culturas contemporáneas. Hacemos esta revisión a partir del trabajo realizado por el grupo de investigación que, desde hace ya cuarenta años, sigue de cerca el desarrollo de este campo interdisciplinar entre arquitectura y educación, a partir de proyectos de investigación, congresos internacionales y revistas internacionales. MÉTODO. Abordar la relación entre arquitectura y educación requiere una combinación metodológica de diferentes disciplinas, como los métodos de la antropología cognitiva, la etnometodología, las ciencias cognitivas, la historia del arte, etc. En el artículo presentamos algunos ejemplos de este cruce interdisciplinar. RESULTADOS. Se demuestra que la relación entre arquitectura y educación aporta soluciones a la necesaria interrelación entre disciplinas actualmente tan alejadas como son la teoría de la arquitectura y del urbanismo, las ciencias sociales y las ciencias cognitivas contemporáneas. DISCUSIÓN. Las conclusiones de esta breve revisión tienen como objetivo resaltar la hipótesis fundamental del artículo, es decir, que sin esta transformación en profundidad de la educación, con el fin de fortalecer la capacidad de las sociedades a defender sus culturas desde el diseño arquitectónico y urbanístico, no podremos conseguir que edificios y ciudades sean de verdad unas infraestructuras sociales y humanas que apoyen la salud física, mental y social de sus habitantes. De esta forma, urbanismo y educación son las dos caras de una misma moneda: cuando una de ellas desaparece, la moneda pierde todo su valor.

Palabras clave: Arquitectura, Educación, Conocimiento social, Ciencia y sociedad. 


\section{Introducción}

La publicación monográfica Arquitectura 63, realizada por los estudiantes del último año de carrera de Arquitectura en Barcelona, para pagarse el viaje de fin de curso a los Estados Unidos (1963), marca un punto de inicio en la investigación sobre arquitectura y educación. En esta publicación, subvencionada en parte por el Colegio de Arquitectos de Cataluña, participaron gratuitamente: W. Gropius, N. Pevsner, L. Mumford, A. Sartoris, B. Zevi, R. Banham, etc., conscientes de la difícil situación política de la España franquista, y también interesados por no perder el contacto con las nuevas generaciones de arquitectos.

En las conclusiones, ya se anunciaba el interés de conectar la arquitectura y el urbanismo con la educación, la investigación y los últimos descubrimientos artísticos, científicos y políticos, para evitar el aislamiento de los arquitectos en un mundo virtual alejado de las necesidades sociales, tal y como los prestigiosos autores de los artículos nos aconsejaban.

Pasados más de cincuenta años, el análisis de las conexiones entre la educación del arquitecto y la arquitectura de la educación sigue su curso. Muchas de las predicciones de aquellos viejos protagonistas en parte se han cumplido, muchos factores, como el uso del ordenador, han modificado aquellas conexiones y han creado nuevas necesidades. Este escrito vale, de forma muy resumida, para interpretarlas.

\section{Un pasado no muy optimista}

En un artículo reciente publicado por la excelente nueva revista del grupo interdisciplinar de urbanistas Degli Territorialisti, de la Universidad de Florencia (Muntañola y Saura, 2014) y gracias al intercambio de correspondencia entre Josep Muntañola y el urbanista norteamericano Lewis Mumford que allá se publican, es posible entender la complejidad de las relaciones históricas y sociales entre arquitectura, urbanismo y ciencias de la educación en los últimos cien años.

Utilizando el símil de la película de I. Bergman, El huevo de la serpiente (1977), referido al origen del fascismo, totalmente comprometido con la defensa de un urbanismo y una arquitectura sin sensibilidad social, implicándose muchos industriales norteamericanos en ello sin que nadie, o muy pocos, como Lewis Mumford, se atrevieran a criticar el proceso. El artículo pone de manifiesto que este autor, reconocido hoy como preecologista, como padre de un ecologismo inteligente, debería ser también reconocido como padre de una arquitectura y un urbanismo responsables capaces de escapar de las garras de la especulación financiera como único motor de crecimiento espacial y tecnológico.

Por lo tanto, el desafío no es tanto el "cambio climático", sino el "cambio social"; y si el "equilibrio ecológico" es ya un desafío fundamental, el "equilibrio social", como veremos, lo es todavía mucho más. Un buen punto de partida es el manifiesto de un grupo de arquitectos y pedagogos de uno de los países pioneros en la pedagogía moderna como es Finlandia (diagrama 1). Aquí se exponen de forma sencilla, condensada y global todas las ideas fundamentales de Lewis Mumford y Alvar Aalto de su amigo y apreciado arquitecto finlandés Alvar Aalto.

La educación tiene una dimensión espacial totalmente ignorada por las mismas razones teóricas que han hecho difícil que la educación del arquitecto en el siglo XXI se encuentre a la altura esperada (Muntañola y Saura, 2005). Por estas mismas razones, las consecuencias del diagrama 2, que relaciona las teorías urbanísticas con las escuelas pedagógicas, han estado totalmente olvidadas, así como las obvias relaciones entre el fascismo y algunas escuelas de arquitectura en la Alemania nazi... ${ }^{1}$. Defendiendo posturas ambiguas no siempre transparentes, al igual que ocurre entre Le Corbusier y el gobierno de Vichy. 
Diagrama 1. Manifiesto PLAYCE

Aproximación filosófica y teórica
a la educación arquitectónica

\section{Diagrama 2. Teorías pedagógicas y teorías urbanísticas}

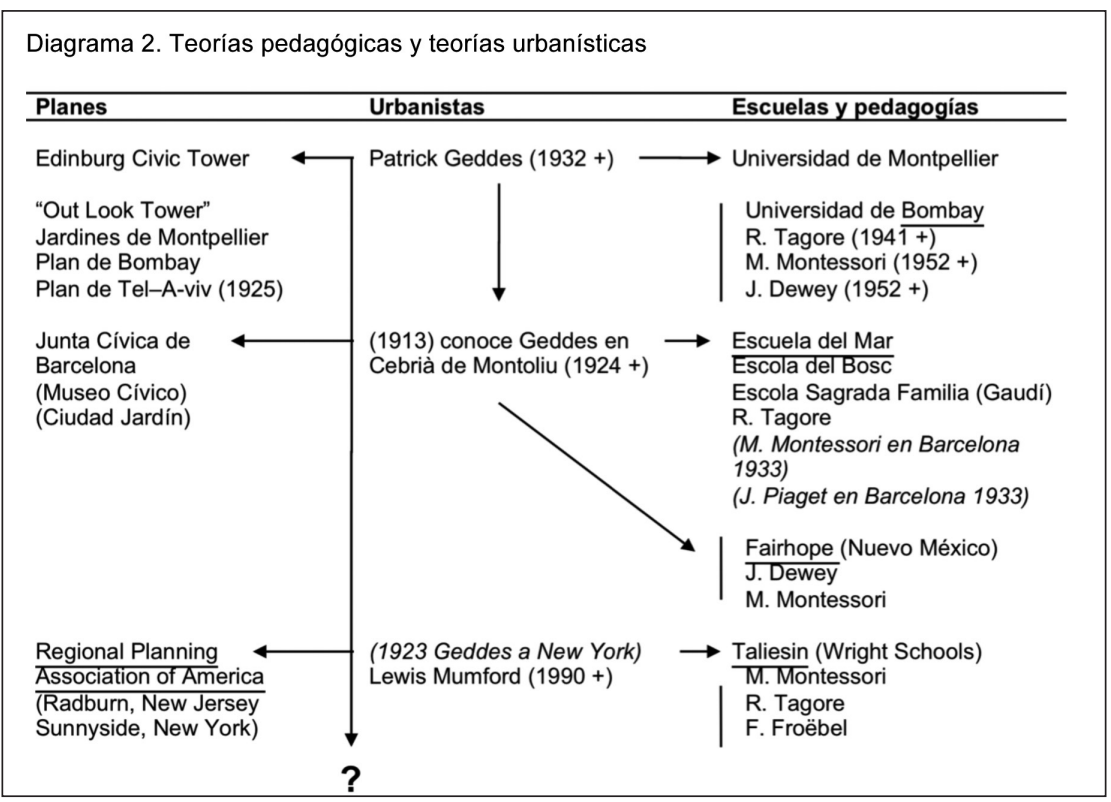

Por otro lado, acabamos de salir de una crisis hipotecaria mundial, originada justamente por el uso financiero abusivo de la arquitectura y del urbanismo, en España y muchos países, pero no ha cambiado la percepción de que no se puede apoyar impunemente un enriquecimiento económico-social, en la especulación abusiva del precio del suelo y de la construcción. El cambio climático da miedo, sin embargo, el cambio social que acompaña el urbanístico y arquitectónico no. Pero quizá deberíamos decir "todavía no", porque, de hecho, el miedo al 
terrorismo ya actúa como fortísimo elemento de discriminación para encontrar trabajo. Se detectó una práctica habitual en las entrevistas de trabajo en las que se exigía información sobre el distrito de residencia a jóvenes franceses, a los que se discriminaba por haber nacido en los barrios periféricos de París, sin tener datos científicos que justificasen la asociación a dicho comportamiento. Difícil encontrar un mejor ejemplo de que algo va mal, muy mal, entre las relaciones entre educación y urbanismo.

Existen programas de actuación excelentes como el de UNICEF-CAI (2009) que mantienen todavía una autonomía frente a las grandes maquinarias financieras y políticas (que están en el origen de la corrupción, por ejemplo), pero se está llegando a la paradoja de poner impuestos al movimiento de capitales, y no frenar el movimiento de las ciudades cubiertas de torres de cien pisos, financiadas por los mismos capitales. Como en el caso de la contaminación: tonto el que no deja de contaminar. Al ser un problema global, la solución debe ser global, a partir evidentemente de propuestas locales muy valientes y muy originales, como la ya citada del movimiento "territorialista" de Florencia.

\section{Hacia una arquitectura y un urbanismo responsables, y cómo educar para conseguirlo}

Volviendo al manifiesto inicial (diagrama 1), no creemos que sus afirmaciones sean "morales" y "políticas", lejanas a planteamientos rigurosamente científicos. Apoyo científico para estas afirmaciones existe y desde todas las disciplinas: antropología cognitiva (D'Andrade, 1995), sociología cognitiva, ciencias cognitivas (Clark y Chalmers, 1998), psicología del desarrollo (Valsiner y Van de Veer, 2000; Langer, Rivera, Schlesinger y Wakeley, 2003), bio-psico-sociología (Piaget y Inhelder, 1967), sociología urbana (Gehl, 1987; Gehl y Gemzoe, 1996; Gehl y Svarre, 2013; Sennett, 2102; Hillier, 1996), teoría de la arquitectura (Pallasmaa, 2006; Holl, 2013; Hillier, 1996), etc. Todos estos estudios dan gran importancia a la interacción entre la educación, la arquitectura y la forma urbana en la calidad de vida de la infancia. Como resumen, véanse los diagramas 3, 4 y 5 sobre nuestro trabajo (Muntañola y Muntanyola, 2012).

Y así llegamos al núcleo fundamental de este artículo: la arquitectura y el urbanismo tienen

\section{Diagrama 3. Ciudades monológicas}

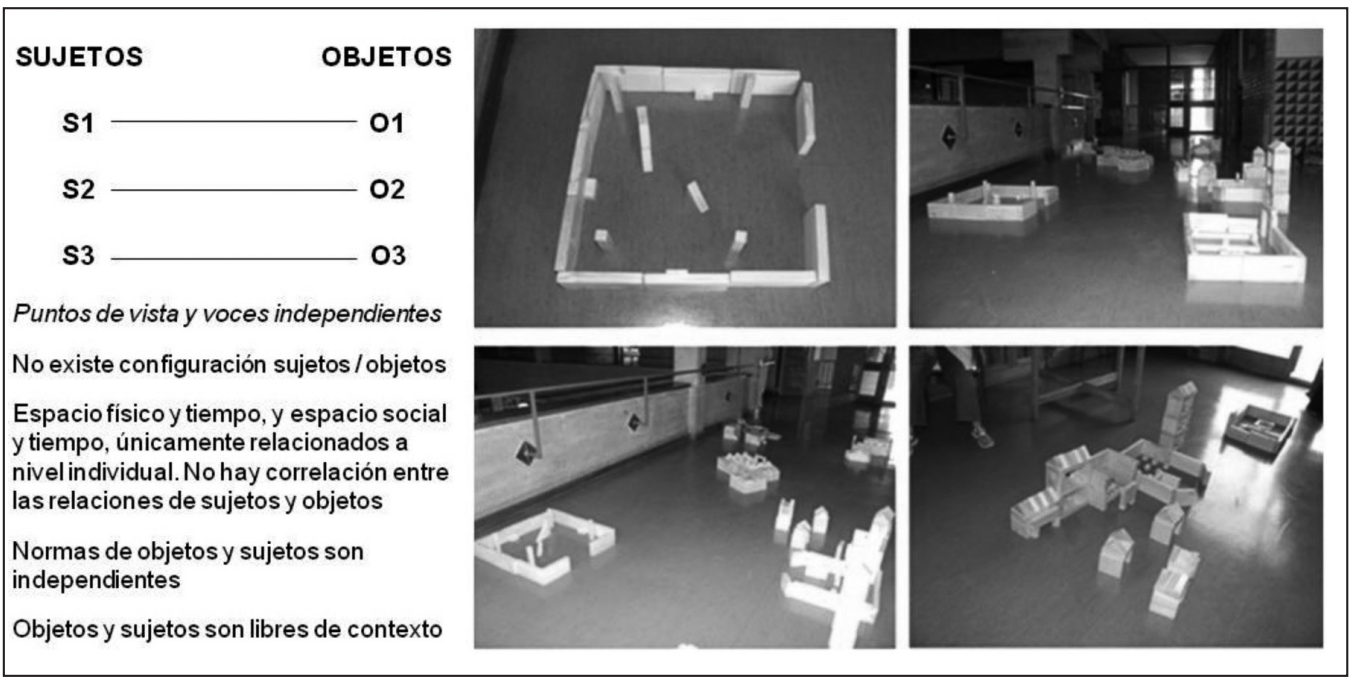




\section{Diagrama 4. Ciudades dialógicas}

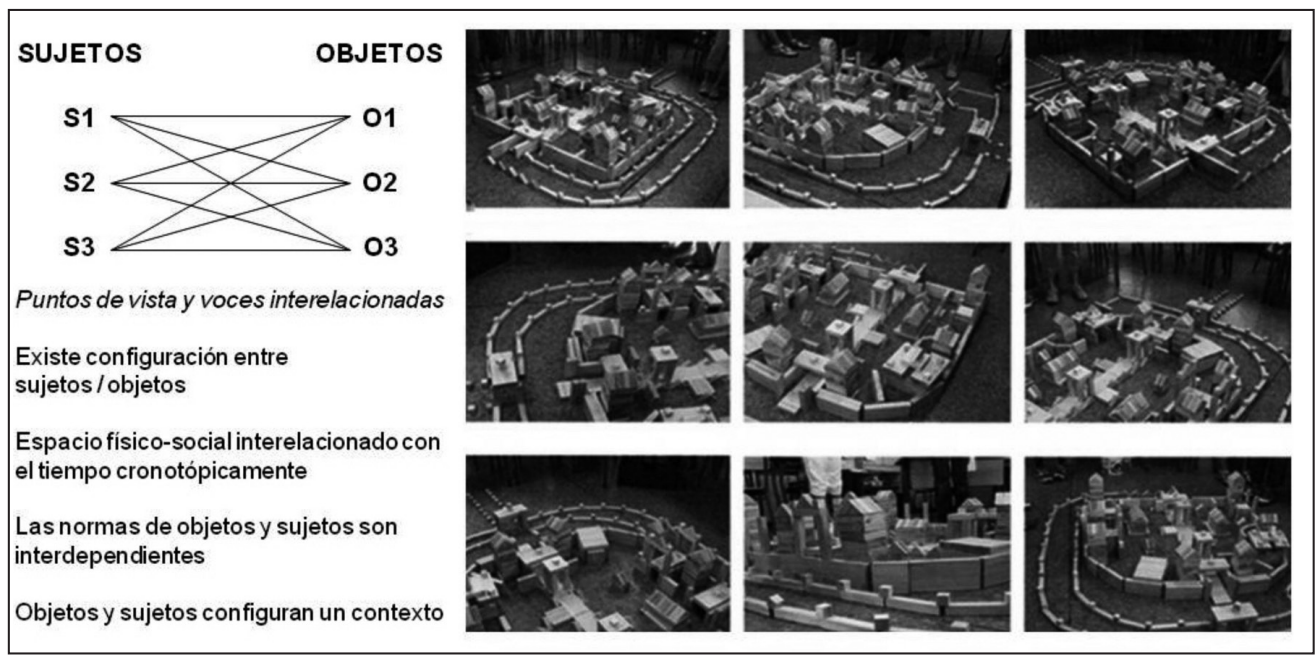

que dejar de ser un puro instrumento técnico y financiero (como un coche o un teléfono), y pasar a ser un instrumento de educación y de transmisión cultural. Una ciudad o un edificio son como los "nidos" de una especie de pájaros. En nuestro caso, los "nidos" son para culturas sociales, no para especies, pero la función del "nido" se mantiene como relación entre el individuo que crece y se educa, y la historia social que se desarrolla (Muntañola, 1980).

El desafío de una arquitectura y un urbanismo local y global es tan gigantesco (más que el climático), que es normal que Lewis Mumford diera miedo en su época y que muchos profesores infantiles no se atrevieran a criticar una decisión urbanística en su municipio, para no perder su puesto de profesor y "la confianza" de su alcalde. No vamos a cambiar el mundo de la política mundial en pocos días. Pero la reciente reunión mundial (febrero, 2015) promovida por el presidente de los Estados Unidos, sobre la prevención mundial del terrorismo (que insistió en no denominar como islámico, ni como algo propio de las clases trabajadoras) es algo interesante.

Por primera vez se planteó directamente que la solución no es el castigo policial, sino la combinación entre educación, arquitectura y democracia social, lo que debe financiarse. Los "nidos" pues, con su huevos, son el objetivo directo.

¿Es lo mismo que el cambio climático? Sí y no

Sí porque incluye la construcción técnico-ecológica y su globalidad / localidad; no, porque incluye sobre una misma base técnico-física una confrontación educativo-dialógica. Sin "nidos democráticos" no hay huevos humanos y sin huevos humanos no hay niños sino solo serpientes. Es, pues, un desafío de enormes dimensiones, el mismo exactamente que planteó Lewis Mumford. Fue acusado de pesimismo, de moralista, de antimoderno, de mal arquitecto, etc. Pero, independientemente de su postura personal, su trabajo iba mucho más allá, anunciaba lo que hoy pasa. A fuerza de hacer ver que todo va bien, cerrando los ojos a los alumnos ante la tecnología y la especulación financiera, dejamos que los "nidos" produzcan huevos de serpiente. Cuando son muchos, nos asustamos, porque, además, las serpientes se comen todos los huevos de los demás. Quizá sea entonces demasiado tarde. 
Pero lo que es muy importante es que el arquitecto o el profesor, que no se atreve a criticar una decisión urbanística y compartidla con sus alumnos, es también responsable, como el director de una multinacional que amenaza a no invertir si no le dejan construir lo que quiere con independencia del impacto social de su obra. Obviamente es el político, y su coste de corrupción, el que debería llevarse la mayor culpa cuando cincuenta años después proliferen las serpientes en sus "nidos", en barrios sin las mínimas condiciones físicas, sociales y culturales: inseguridad, drogadicción, falta de mantenimiento, marginación social, falta de escolarización, etc.

Dada la magnitud enorme del problema, la consciencia individual y colectiva sobre la importancia de la arquitectura y del urbanismo se convierten en indicadores de la necesaria "responsable prevención", esencial para no acabar en marginaciones y frustraciones que serán el caldo de cultivo de actitudes en contra de la civilización.

En experiencias recientes, este año, niños y niñas de Barcelona, tal como descubre magistralmente Mumford en sus escritos sobre cómo se "olía" el fascismo de Hitler en el aire, al construir maquetas de ciudades, es decir "nidos", los niños y niñas, como increíbles olfateadores/as sociales (les va la vida), iban construyendo, tanto en grupo como individualmente, y a la vez, expresando sus sensaciones de qué civilización estaban construyendo: "Yo me voy a California, a Los Ángeles, porque aquí no hay nada que hacer", decía convencidísima una niña de diez años mientras construía con gran delicadeza un barrio, un "nido", con gran afecto y extraordinaria precisión, situado, mentalmente, en aquella ciudad americana, por considerar que en Barcelona aquel "nido" era utópico e imposible. Otros, a los siete años, construían edificios sin puertas, ni ventanas con inmensos ascensores "con códigos de acceso", y sin identidad ninguna, por "si acaso".

El espacio se incorpora a la vida mental, física y social, como la música, la literatura o la danza (Muntañola y Muntanyola, 2011), a partir de las relaciones intersubjetivas pautadas por las diferentes culturas. En pleno siglo XXI, esta incorporación sigue en la oscuridad, como si fuera imposible estudiarla e investigarla. De este modo, el espacio se transforma sin ningún fundamento teórico que legitime esta transformación. Estudios importantes, como los de Pierre Kaufmann (1995) o Bill Hillier (1996), podrían cambiar esta situación, pero su impacto en la arquitectura de la educación ha estado bajo mínimos.

La significación cultural del espacio, tal y como indican los diagramas 5 y 6 , depende del tipo de relaciones intersubjetivas. $\mathrm{Y}$ al revés, cada arquitectura actúa, en negativo, sobre las posibles relaciones intersubjetivas, hasta hacer imposible ciertas relaciones y facilitar más

Diagrama 5. Interacción físico-social en la creación de "Lugares para vivir"

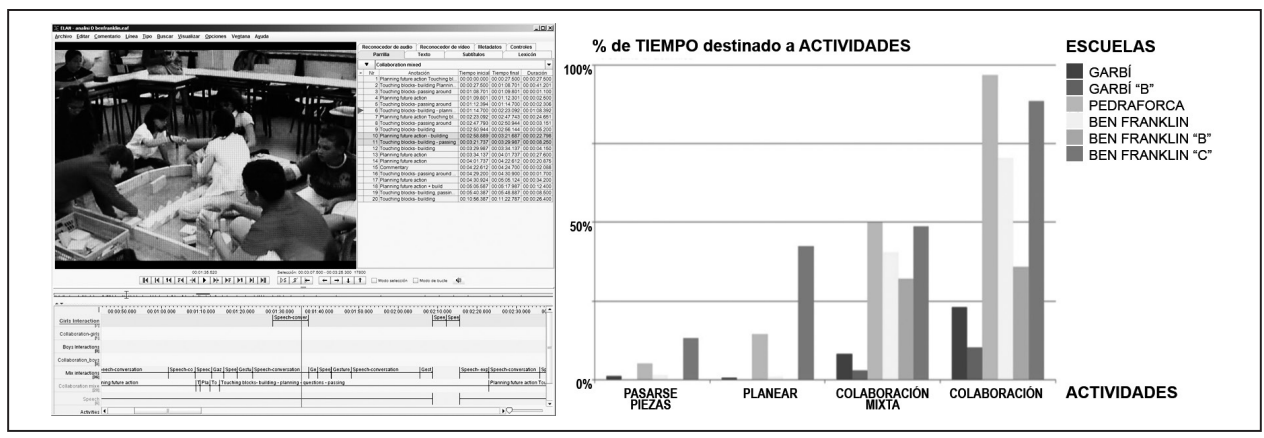


Diagrama 6. Intersubjetividad y actividades escolares

\begin{tabular}{|c|c|c|}
\hline & Ciudad "Monológica" & Ciudad "Dialógica" \\
\hline Número de elementos diferentes & 10 & 60 \\
\hline Participación de & No existe & Alta participación \\
\hline Celebraciones y visitas organizadas & No existen & Muchas \\
\hline Teatro & No existe & Muy importante \\
\hline
\end{tabular}

algunas que otras, sin llegar nunca a una fatal predeterminación del comportamiento a partir de la geometría, o por el propio uso del ordenador. De aquí su influencia "genérica".

Y es por esto que los niños y niñas representan el espacio en relación al tipo de modelo de educación social que reciben, con especial sensibilidad al teatro, a la relación entre géneros (coeducación o no), a la música en grupo y a las relaciones intergeneracionales (con padres, hijos, etc.). En resumen, niños y niñas tratan el espacio como "genérico", en el sentido exacto que E. Husserl define a la geometría, como el punto de encuentro entre la madurez de una sociedad y la madurez educativa del niño. También es en este punto que la obra póstuma de Pierre Kaufmann encuentra el sentido de una arquitectura como una "forma de civilización" contra la barbarie de un fascismo, por ejemplo, en el que la geometría produce los campos de exterminio de judíos, a donde los nazis disfrutaban del dolor de los otros, signo clarísimo de "barbarie" (Kaufmann, 1995).

Si las matemáticas sirven para enseñar informática, física, biogenética, etc., la arquitectura y el urbanismo son insustituibles en la enseñanza de la historia, la arqueología, la ecología urbana, la geografía, etc. No se trata, únicamente, de enseñar estilo, técnicas constructivas y formas urbanas a niños y niñas, sino de hacerlos conscientes de la importancia del espacio para la coexistencia o la marginación social o para la modificación de las relaciones intersubjetivas de género, intergeneracionales o interculturales. La historia de las formas urbanas es aquí esencial, ya que estas llevan escritas las raíces culturales de cada momento histórico como "proyecto". Excelentes, en este punto, las reflexiones de M. Bakhtin sobre Goethe (Bakhtin, 1986).

\section{La esperanza dialógica e interactiva de una pedagogía de la arquitectura, y sus enemigos}

Es el psicosociólogo Aaron Cicourel, residente en San Francisco, el que ha definido hoy, quizá con mayor precisión, lo que está ocurriendo entre la mente y la sociedad con sus conclusiones sobre el Alzheimer en su artículo: "Memoria colectiva: una fusión de mecanismos cognitivos y procesos culturales" (2014).

No nos alejamos del tema aunque lo parezca. Veamos sino el diagrama IV los índices de calidad urbanística que deben tener las Ciudades Amigas de la Infancia, dictados en el "report" anual de la Infancia de Unicef (2012). Estos criterios responden, punto por punto, a las afirmaciones de Aaron Cicourel sobre la necesaria "fusión" entre conocimiento y cultura, y entre individuo y sociedad, para tener una buena memoria (Cicourel, 2014).

Estamos definiendo que la realidad física, base del individuo y la arquitectura y urbanismo, 
también físicos, realizan ambas el papel de mediación a lo que antes hacíamos referencia. Y así entramos en la esperanza dialógica, debida sobre todo al pensador ruso Mijaíl Bajtín, hoy figura mundial, que pasó desapercibido casi hasta su muerte en 1975 (Muntañola, 2006). Fue Bakhtin el que vio lo importante de esta conexión al escuchar una conferencia sobre la "embriogénesis" en 1920 en San Petersburgo y desarrollar inmediatamente sus tesis sobre la estructura cronotópica de la cultura, sea esta hecha de libros, diseños, música o pinturas.

No existe mejor descripción de lo que es un diseño interactivo y dialógico que este texto (Bakhtin, 1981: 252). Muy pocos diseñadores realizan su trabajo desde esta perspectiva de relaciones entre personas a través del diseño de objetos culturales, en nuestro caso, edificios, ciudades o paisajes dialógicos, culturales e interactivos. Muchos se contentan con diseñarse a sí mismos una y otra vez, y en repetir una y otra vez las mismas formas en cualquier lugar y circunstancia, presumiendo de la cultura de los espacios potenciales y de las condiciones locales de los edificios.

Obras fundamentales sobre una nueva pedagogía han sido, en estos cincuenta años, las de Spiro Kostof (1977), Martha Pollack (1997), Bill Hillier (1996) y Lewis Mumford (1922), a los que hemos referenciado en centenares de publicaciones (Muntañola, 2004). Lewis Mumford (1945) y M. J. Abercrombie (1960) escribieron los fundamentos de una nueva pedagogía del diseño conectando la arquitectura con los avances más significativos en el campo de las ciencias humanas y de la filosofía de las ciencias, tal como E. S. Casey (1997), P. Ricoeur (2003) y P. Kaufmann (1967, 1995) investigaron, conjuntamente con muchos otros pensadores del siglo XX.

Finalmente, y en relación al uso del ordenador, el impacto de las recientes escuelas "cognitivas" del pensamiento "distribuido", "corporizado" y "extendido" ha abierto enormes perspectivas en la educación del arquitecto, y en la educación en general, como veremos.

Tomando como ejemplo el concepto ya citado de la cultura "cronotópica", construido por M. Bajtín (1990), y las tres dimensiones de la cultura del hombre, definidas por E. Hutchins, que se corresponden, punto por punto, con el esquema cronotópico del propio Bakhtin, podemos ya darnos cuenta del enorme futuro de una pedagogía fundamental del arquitecto en el cruce de los tres ejes del diagrama 7 , que no es más que una visión contemporánea de la vieja estructura teórica de la arquitectura según Vitruvio (y refundada por Heidegger) entre: "proyectar, construir y habitar". A pesar del antiguo origen del tema, estamos delante de una revolución en la educación del arquitecto. Pero como estamos intentando averiguar recientemente (Muntañola, 2011), es Bakhtin el que, con una crítica muy dura contra E. Kant, E. Saussure y, de paso, contra M. Heidegger, pone el dedo en los aspectos fundamentales del problema.

La pregunta es: ¿por qué no se ha notado el impacto de la estructura cronotópica básica en las pedagogías de educación del arquitecto, a pesar de que la armonización ética, estética y científica, entre proyectar, construir y habitar, parece una teoría muy valiosa y una manera contemporánea de reactivar la sabiduría del arquitecto? (Bakhtin, 1981). Tal y como analizan las publicaciones mencionadas de S. Kostof (1977), M. Pollack (1997), B. Hillier (1996), etc., las escuelas de arquitectura que, en la valoración de proyectos siempre han intentado encontrar una síntesis cronotópica entre arte, ciencia y política, a nivel teórico, han encontrado enormes dificultades en dicha síntesis, haciendo prácticamente inexistente la investigación en la profesión de arquitectura, tal como ha explicado con mucha claridad J. Till en un reciente estudio (2012).

La respuesta debe buscarse en unos errores teóricos que han enraizado fuertemente en la pedagogía del arquitecto y que costará destruir, 
Diagrama 7. El cronotopo creativo

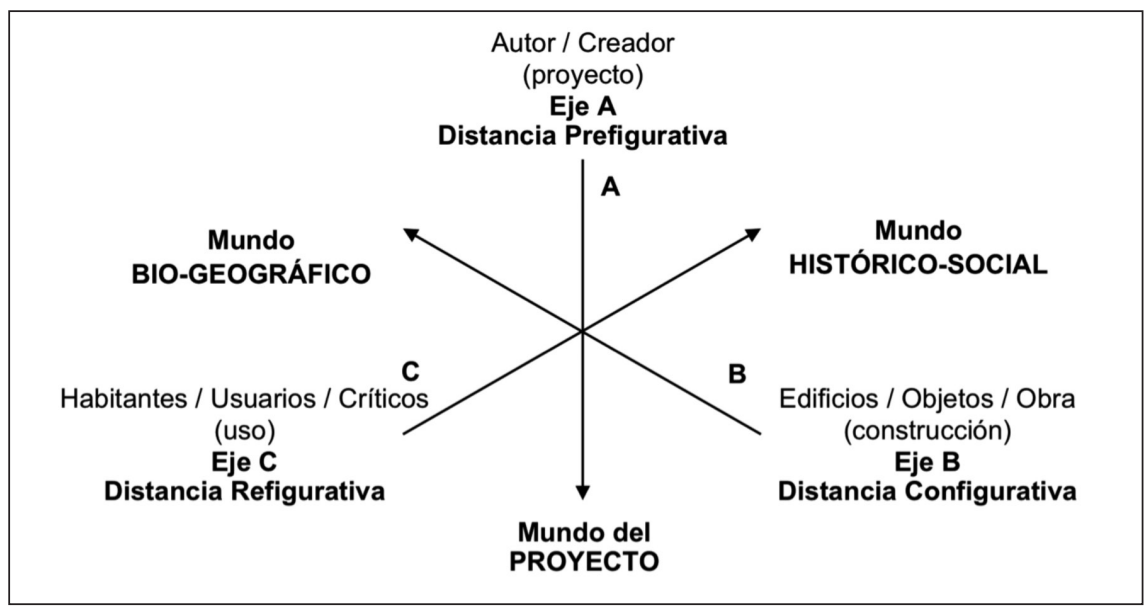

porque esta destrucción modificaría la profesión. Algunos de estos errores son los siguientes:

- Considerar que la abstracción no tiene nada que ver con la vida real. Como consecuencia, un edificio o una ciudad, tienen una significación con independencia del contexto histórico-geográfico en el que existen.

- Considerar que la imaginación proyectual del arquitecto depende de su cerebro único e intransferible, no de un diálogo social. Por tanto, el arquitecto no tiene responsabilidad sobre el uso de sus edificios.

- Considerar que la técnica constructiva responde exclusivamente a criterios científicos. El arte de construir o la ética del construir son aspectos secundarios, que no se pueden articular con las leyes científicas y disciplinarias de la construcción.

Todos estos errores tienen como consecuencia que la arquitectura y el urbanismo responden a una "disciplina" específica, autónoma y diferente de cualquier conocimiento fuera de la propia profesión, como advierte J. Till (2012).

De esta manera, las expectativas de una revolución dialógica en la enseñanza de la arquitectura podrían desvanecerse, y las conexiones entre la arquitectura y el urbanismo y las nuevas teorías filosóficas, de las ciencias sociales y de las ciencias cognitivas se desvanecerían también.

El motor de esta revolución ha estado, y aún lo es, la profunda articulación entre la geometría, la construcción y el uso social, intersubjetivo del espacio. Esta articulación está perfectamente definida en los escritos de E. Husserl, especialmente en la traducción al francés del Origen de la geometría, con una larguísima introducción realizada por un, entonces desconocido, Jacques Derrida (Husserl, 1962).

En su escrito original en alemán, en el año 1936, Husserl determina el origen social, histórico e intersubjetivo de la geometría. Es exactamente, un origen "genérico" allí donde la madurez de la sociedad y la del niño se articulan en una verdad que va más allá de la invención de una única persona aislada. La conexión con el mundo de la educación es, entonces, clarísima, y las relaciones con las otras perspectivas en las ciencias sociales, abiertas por M. Bakhtiny E. Hutchins, evidentes. También las indagaciones de B. Hillier, P. Ricoeur y P. Kaufmann han reafirmado las intuiciones de Husserl. Pero, muchos arquitectos y muchos profesores de primaria, piensan aún que la 
geometría es simplemente, solamente una técnica, e ignoran sus dimensiones teóricas, estéticas, históricas y ético-políticas (Laaksonen y Räsänen, 2006: 97).

En estas condiciones, la educación del arquitecto no puede evolucionar correctamente, ya que, desconectada de la investigación contemporánea en las artes, las ciencias, etc., no puede hacer otra cosa que repetir los mismos errores teóricos y las mismas formas, sin nunca reconocer el origen social de ideas, emociones, formas, símbolos y/o comportamientos. Y es una lástima, porque, como puede comprobarse en el centenar de tesis doctorales leídas entre 1985 y 2013 en el grupo de investigación GIRASUPC, los arquitectos pueden hacer aportaciones específicas en el mundo de la investigación que las otras profesiones no pueden realizar (ver diagramas 8 y 9).

\section{Diagrama 8. La investigación en arquitectura: las 7 ramas temáticas}

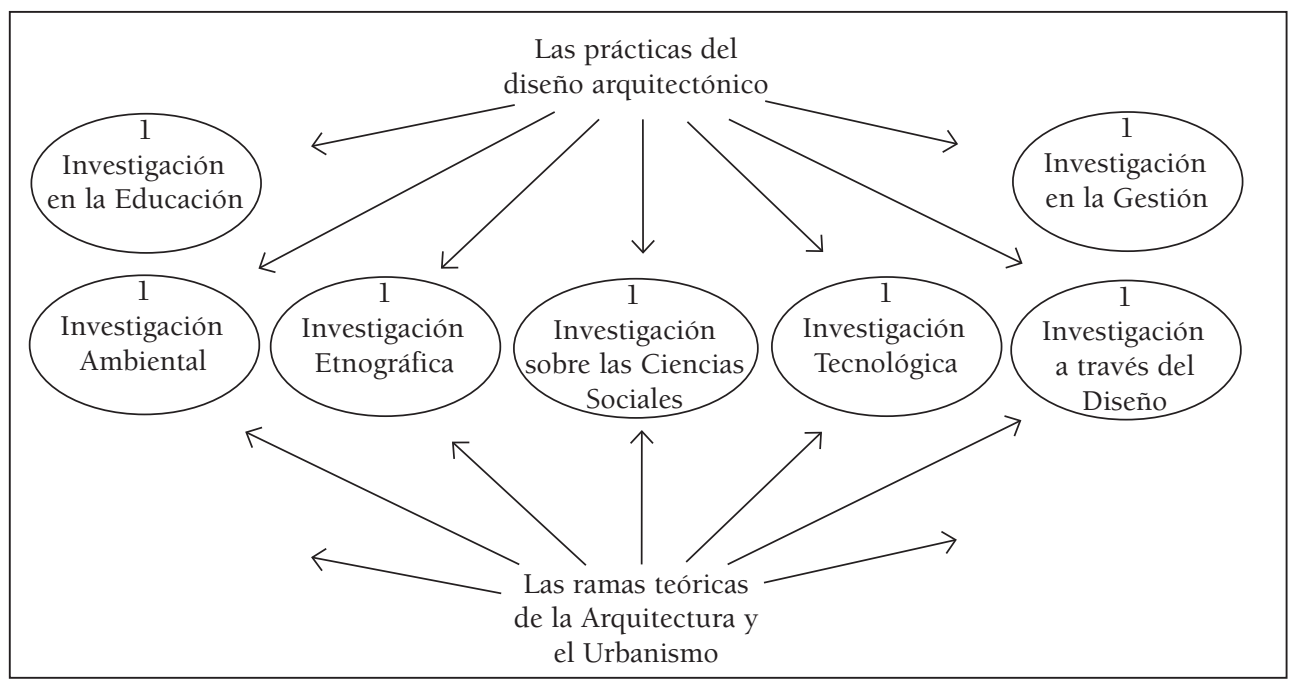

DiAgrama 9. La investigación en arquitectura: las 3 ramas teóricas

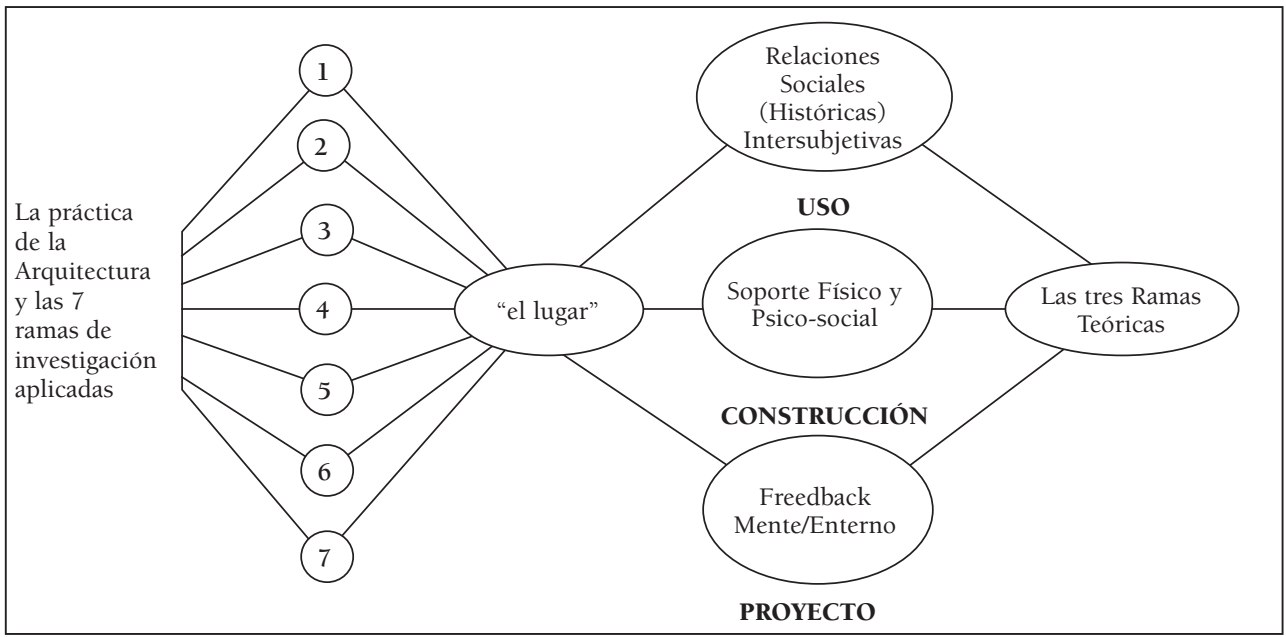


Diagrama 10. Patología cognitivo-espaciales

\begin{tabular}{|l|c|l|}
\hline \multicolumn{1}{|c|}{ Patologías Niños Salvajes } & $\begin{array}{c}\text { Patologías de niños y niñas } \\
\text { con exceso de } \\
\text { virtualización }\end{array}$ & $\begin{array}{c}\text { Aspectos Arquitectónicos } \\
\text { (psico-sociales) }\end{array}$ \\
\hline $\begin{array}{l}\text { Movimientos desordenados, } \\
\text { caminar como un lobo, etc. } \\
\text { Posturas extrañas }\end{array}$ & $\begin{array}{c}\text { Accidentes por falta de } \\
\text { experiencia en el mundo } \\
\text { físico. Accidentes. }\end{array}$ & $\begin{array}{l}\text { Movimientos humanos } \\
\text { correctos (psico-social) }\end{array}$ \\
\hline $\begin{array}{l}\text { No reconocer su propia } \\
\text { imagen en un espejo }\end{array}$ & $\begin{array}{l}\text { Confusión mundo virtual y } \\
\text { real: simbolismos que no } \\
\text { funcionen en el mundo real }\end{array}$ & $\begin{array}{l}\text { Reconocimiento correcto de } \\
\text { la propia imagen e identidad } \\
\text { en el medio construido. }\end{array}$ \\
\hline $\begin{array}{l}\text { Ausencia de identidad sexual } \\
\text { y de relaciones sexuales } \\
\text { normales }\end{array}$ & $\begin{array}{l}\text { Relaciones intersubjetivas en } \\
\text { relación al sexo difíciles o } \\
\text { inexistentes (Japón) }\end{array}$ & $\begin{array}{l}\text { Inter-subjetividad sexual } \\
\text { activa y saludable (identidad } \\
\text { psico-social) }\end{array}$ \\
\hline $\begin{array}{l}\text { No hablan, ni dibujan, ni } \\
\text { hacen mímica, etc. } \\
\text { correctamente }\end{array}$ & $\begin{array}{c}\text { Pautes artísticas en el mundo } \\
\text { virtual pero sin una } \\
\text { socialización intersubjetiva } \\
\text { fuera de "internet" }\end{array}$ & $\begin{array}{l}\text { Comunicación geométrica, } \\
\text { musical y lingüística }\end{array}$ \\
\hline $\begin{array}{l}\text { Ritmos de noche y día de } \\
\text { otras especies no humanas } \\
\text { Descontrol emocional }\end{array}$ & $\begin{array}{l}\text { Stress, descontrol emocional } \\
\text { por no seguir el mundo real, } \\
\text { reglas del mundo virtual. } \\
\text { Alteración del sueño. }\end{array}$ & $\begin{array}{l}\text { Ritmo emotivo, noche y día, } \\
\text { sueños }\end{array}$ \\
\hline
\end{tabular}

Damos por supuesto que se sabe que el desarrollo de los niños y niñas sigue unas etapas, desde el punto de vista de la génesis de la arquitectura y un urbanismo, basados desde una topología sensorio-motora (Muntañola, 2013) a una topología matemática, base del ordenador y del mundo digital de hoy (Muntañola, 2008), pasando por la arquitectura y el urbanismo de Euclides y la geometría proyectiva. En otras publicaciones hemos descrito todas estas etapas ampliamente.

Existen manuales concretos (Muntañola, 1985) y estamos confeccionando nuevos manuales (Muntañola, 2016) poniendo al día ejemplos y actividades. Pero lo más importante, insistimos, es la conexión entre arquitectura, urbanismo e interacción social, fundamento de cualquier relación entre arquitectura y educación.

\section{Conclusión}

Contra lo que algunos lectores puedan pensar, todo este planteamiento no es incompatible con la pedagogía de una arquitectura puramente economicista o estrictamente determinada por códigos científicos. No comparto estas pedagogías, pero estoy de acuerdo con Jeremy Till (2012) en que estos ejemplos de arquitectura "comercial" pueden ser la base de buenas investigaciones, y están determinadas por estructuras cronotópicas específicas que pueden ser interesantes para una análisis general de arquitectura y urbanismo en el siglo XX.

Es necesario definir cuáles son los valores cronotópicos que, en cada caso, determinan las cualidades de un producto, por ejemplo, la medida física, el precio de mercado, etc., más allá de cualquier otra determinación. Esto solo ya puede ser una investigación significativa.

Pero analizar innovaciones más allá del precio por metro cuadrado es también una tarea insubstituible para comprender el papel cultural del espacio al que se está haciendo referencia. Esta tarea proto-arquitectónica es la que algunos arquitectos han empezado a llevar a 
cabo: J. Pallasmaa, S. Holl y muchos otros se están tomando con mucha seriedad esta posibilidad de relacionar la educación del arquitecto con la arquitectura de la educación (Holl, 2013), pero esto nos llevará a otro artículo en un futuro cercano.

Finalizando, como hemos anteriormente planteado, la educación de la arquitectura y la arquitectura de la educación son dos caras de la misma moneda. Análogamente (ver diagrama 2), urbanismo y educación son los dos extremos de una cadena que permite la supervivencia de alguna civilización. Cuando esta cadena se rompe, la civilización se convierte en Barbarie.

Es quizá, el filósofo francés Pierre Kaufmann quien definió claramente las diferencias entre un urbanismo civilizado y la barbarie, poniendo como ejemplo de barbarie los campos de concentración para eliminar judíos, homosexuales, presos políticos, etc.

Por otro lado, los niños salvajes (los salvajes, no los de tribus primitivas), con su soledad como individuos que crecen entre cabras, osos o lobos, manifiestan síntomas muy alejados de los niños "educados". Si relacionamos todos estos diagramas, del I al VII, tendremos una vista panorámica sobre la enorme responsabilidad ética de los arquitectos y urbanistas, ya anunciada por Aristóteles hace miles de años, cuando situó la sabiduría del arquitecto como el modelo de la sabiduría que necesita el legislador y el educador. Es decir: para que cambie la educación del arquitecto, es necesario que cambie la arquitectura de la educación. Esto implica que la arquitectura y el urbanismo se impartan en escuelas primarias con la misma seriedad que las matemáticas, las lenguas o la filosofía.

En estos cincuenta años, mucho trabajo se ha llevado a cabo en este sentido, pero mucho trabajo está todavía por hacer (Muntañola, 2007; Muntañola y Muntanyola, 2012). Las razones del retraso son las mismas en educación que en arquitectura, a pesar de que toda la investigación demuestre las relaciones estrechas entre las patologías sociales y las arquitecturas que las facilitan (diagrama 10), o la clara relación entre urbanismo y arquitectura y educación social y salud integral de niños y niñas en el mundo urbano (diagramas $3,4$ y 5$)$.

\section{Notas}

* Con el apoyo del Ministerio de Economía y Competitividad. Proyecto EDU 2013-41328-P.

${ }^{1}$ Únicamente Lewis Mumford se atrevió a documentar las conexiones con Hitler de algunos arquitectos de la Bauhaus (ver Grupo Circle, a Londres). Después de la guerra, en Estados Unidos, se silenció la cuestión.

${ }^{2}$ ver el apartado "Tesis" de la web: www.arquitectonics.com

\section{Referencias bibliográficas}

Abercrombie, M. L. (1960). The Anatomy of Judgement: An Investigation into the Processes of Perception and Reasoning. London: Hutchinson.

Bakhtin, M. (1981). The Dialogical Imagination by M.M. Bakhtin (M. Holquist, ed.). Austin: University of Texas Press.

Bakhtin, M. (1986). Speech Genres and Other Late Essays. University of Texas Press.

Bakhtin, M. (1990). Art and Answerability: Early Philosophical Essays. University of Texas Press. 
Casey, E. S. (1997). The Fate of Place. Berkeley: California Press.

Cicourel, A. V. (2014). Colective Memory, A Fusion of Cognitive Mechanisms and Cultural Processes. Revue de synthèse, 135 (6).

Clark, A., y Chalmers, D. (1998). The extended mind. Analysis, 58, 7-19.

D'Andrade, R. G. (1995). The Development of Cognitive Anthropology. Cambridge: Cambridge University Press.

Escuela Técnica Superior de Arquitectura de Barcelona (1963). Arquitectura 63: Publicación de la ETS de Arquitectura de Barcelona, que ha iniciado la 89 promoción, en conmemoración de la VIII Conferencia internacional de estudiantes de Arquitectura. Barcelona: ETS de Arquitectura.

Gehl, J. (1987). Life between buildings: Using public space. Copenhagen: Arkitektens Forlag. Gehl, J., y Gemzoe, L. (1996). Public Spaces Public Life. Copenhagen: The Danish Architectural Press. Gehl, J., y Svarre, B. (2013). How to study public life. Washington DC: Island Press.

Hillier, B. (1996). Space is the Machine: A configurational theory of Architecture. Cambridge: Cambridge University Press.

Holl, S. (2013). Steven Holl: The World We Live In. Urban Environment Design Mazaginze, 72.

Husserl, E. (1962). L'origine de la géométrie. Presses universitaires de France.

Kaufmann, P. (1995). Qu'est-ce Qu’un civilisé? Cahors, France: Atelier Alpha.

Kaufmann, P. (1967). L'experience emmocionnelle de l'espace. París: Vrin.

Kostof, S. (ed.) (1977). The Architect: Chapters in the History of the Profession. Oxford: Oxford University Press.

Laaksonen, E., y Räsänen, J. (eds.) (2006). PLAYCE: Architectura Education for Children and Young People. Helsinki: Alvar Aalto Academy.

Langer, J., Rivera, S., Schlesinger, M., y Wakeley, A. (2003). Early Cognitive Development: Ontogeny and Phylogeny. En J. Valsiner y K. Connolly (eds.), Handbook of Development and Psychology (pp. 141-172). London: Sage.

Mumford, L. (1922). The Story of Utopias. New York: Boni and Liveright.

Mumford, L. (1945). La cultura de las ciudades. Buenos Aires: Emecé.

Muntañola, J. (1980). Towards and Epistemological Analysis of Architectural Design as a PlaceMaking Activity. En G. Broadvent, T. Llorens y R. Bunt (eds.), Behavior and Meaning in the Built Environment. London: Wiley and Sons.

Muntañola, J. (1985). Comprendre l'Arquitectura. Barcelona: Editorial Teide-Barcelona.

Muntañola, J. (2004). Arquitectura, Educación y Dialogía Social. Revista Española de Pedagogía, 228, 221-228.

Muntañola, J. (ed.). (2006). Arquitectura y Dialogía. Arquitectonics: Mind, Land E Society (vol. 13). Barcelona: Iniciativa Digital Politècnica.

Muntañola, J. (2007). Las formas del tiempo: Arquitectura, Educación y Sociedad (vol. 1). Badajoz: Abecedario.

Muntañola, J. (2008). Architecture On The Threshold Of The Digital Age: Revolution Or Regression? En H. Heyen y A. Rauta (eds.), EAAE Transactions on Architectural Education - Proceedings of four EAAE-ENHSA Subnetwork Workshops on Architectural Theory (vol. 43). Copenhagen: EAAE-ENHSA.

Muntañola, J. (ed.). (2016). Cómo educar con la arquitectura desde la infancia. Arquitectonics: Mind, Land E Society ((en proceso de publicación) vol. 31). Iniciativa Digital Politécnica.

Muntañola, J., y Muntanyola, D. (2011). La sociología del espacio al encuentro de una Arquitectura oculta en la Educación. Revista Española de Sociología de la Educación , 4 (2), 133-151.

Muntañola, J., y Muntanyola, D. (2012). La arquitectura desde lo salvaje. In Arquitectura e Investigacion. Arquitectonics: Mind, Land E Society (vol. 24, pp. 35-38). Barcelona: Edicions UPC. 
Muntañola, J., y Saura, M. (2005). On the Search of the Research Core of Architecture. In C. Spiridonidis (Ed.), Ideas and Reflections on Architectural and Urban Design Education. EAAE Transaction on Architectural Education (vol. 28, pp. 148-151). Thessaloniki, Greece: European Network of Schools of Architecture.

Muntañola, J., y Saura, M. (2011). Bakhtin, Architectonics and Architecture. The XIV Bakhtin Conference, (pp. 55-56). Bologna.

Muntañola, J., y Saura, M. (2013). La investigación proyectual a examen: un gran desafí a la Arquitectura del Siglo XXI. Caracas: Ediciones Facultad de Arquitectura y Urbanismo, Universidad Central de Venezuela. Colección Ensayos de Postgrado.

Muntañola, J., y Saura, M. (2014). On the search of a lost modern planning: Throughout the legacy of Lewis Mumford. Scienza del Territorio, 2, 433-444.

Pallasmaa, J. (2006). Los ojos de la piel: la arquitectura y los sentidos.Barcelona: Gustavo Gili.

Piaget, J., y Inhelder, B. (1967). The Child's Conception of Space. New York: Norton.

Pollack, M. (ed.). (1997). The Education of the Architect: Historiography, Urbanism, and the Growth of Architectural Knowledge. Cambridge, Mass: MIT Press.

Ricoeur, P. (2003). Arquitectura y Narratividad. Arquitectura y Hermenéutica. Arquitectonics: Mind, Land y Society, 4, 9-30.

Sennett, R. (2012). Together: The Rituals, Pleasures and Politics of Cooperation. Yale: University Press. Till, J. (2012). ¿Qué es investigar en arquitectura? Investigar en arquitectura: tres mitos y un modelo. Arquitectura e Investigación. Arquitectonics: Mind, Land E Society, 24, 13-20.

UNICEF (2012). The Urban Child. World Report, New York.

Unicef-Ciudades Amigas de la Infancia (2009). Indicadores municipales de aplicación de la Convención sobre los Derechos del Niño. Una herramienta para la elaboración de informes de situación de la infancia en el ámbito local español.UNICEF.

Valsiner, J., y Van de Veer, R. (2000). The Social Mind. Cambridge: Cambridge University Press.

\begin{abstract}
From the education of the architect to the architecture of education: an essential dialogue
\end{abstract}

INTRODUCTION. The present article is a review of the relationships between architecture and education, not from a perspective that insists only on the presence of the architecture and urban planning in the educational contemporary curricula, following the much imitated case of Finland, but from a point of view that considers the whole education changed by the spatial dimensions of culture. We start from the work carried out by the research group working into education on architecture during the last 40 years, thanks to developmental interdisciplinary research projects, international congresses and international journals. METHOD. Addressing the relationship between architecture and education methodological approaches requires a combination of different disciplines, such as methods from: Cognitive Anthropology, Ethnomethodology, Cognitive Sciences, History of Art, etc. In the present article we describe some examples of this interdisciplinary crossroads. RESULTS. The article shows that the relationship between architecture and education provides solutions to the interrelations between disciplines described above that have been isolated until now. DISCUSSION. The findings of this brief revision aim to highlight the fundamental hypothesis of the article, that is to say that without these deep changes in education, with the aim to strengthen the capacity of the 
societies to defend their cultures with good architecture and urban design able to support physical, mental and social health of their inhabitants. In this way, urban planning and education are the two faces of the same coin: when one of them disappear the currency loses its full value.

Keywords: Architecture, Education, Social Knowledge, Science and Society.

\section{Rèsumè}

De l'Éducation de l'Architecte, jusqu'à l'architecture de l'Éducation: un dialogue essentiel

INTRODUCTION. Cet article analyse les relations entre l'architecture et l'éducation mais, pas seulement des une perspective qui mette l'accent sur la présence de l'architecture et l'urbanisme dans les programmes scolaires, comme ce le cas dans des pays comme Finlande, mais aussi des une perspective nouvelle qui appuie la transformation de l'éducation à travers des dimensions sociales et physiques propres de l'architecture et l'urbanisme. Comme groupe de recherche interdisciplinaire, nous possédons 40 années d'expérience sur ce sujet. MÉTHODE. Analyser les relations entre l'architecture et l'éducation exige d'une vision interdisciplinaire avec plusieurs perspectives apportées par l'anthropologie cognitive, l'ethnométhodologie sociale et l'histoire de l'art. Nous présentons ici des exemples de cette pluralité. RÉSULTATS. Nous pouvons déduire que les relations entre l'architecture et l'éducation démontrent que les théories de l'architecture et de l'urbanisme, les sciences sociales et les sciences cognitives ont des relations significatives dans le cadre des sociétés contemporaines. DISCUSSION. Les conclusions de cet article ont pour finalité fondamental mettre en relief que l'éducation est essentielle pour arriver à une culture capable de défendre et construire une véritable culture social à partir des interventions architectoniques et urbaines qui soutiennent la santé physique, mental et social de ses habitants. Ainsi, l'architecture et l'urbanisme sont les deux faces d'une même médaille et quand une d'elles disparaît, cette médaille perd toute sa valeur.

Mots clés: Architecture, Éducation, Connaissance Social, Science et Société.

\section{Perfil profesional de los autores}

\section{Magda Saura Carulla}

Profesora coordinadora del Grupo GIRAS. Graduada en Arquitectura por la Universidad de California en Berkeley (BA-1974, PhD-1988) y doctora en Historia de la Arquitectura por la Universidad Autónoma de Barcelona. Premio FAD (Mención Especial) 1994, autora del proyecto del Plan especial de protección del frente marítimo de Empúries 1990-1992 (Saura y Muntañola, Asociados). Autora de libros y artículos sobre la evaluación del impacto ambiental. Ver web: www.arquitectonics.com

Correo electrónico de contacto: magdalena.saura@upc.edu 


\section{Josep Muntañola Thornberg (autor de contacto)}

Profesor sénior Universitat Politècnica de Catalunya. Grupo GIRAS. Director de la Escuela de Arquitectura de Barcelona, 1980-1984. Director del Departamento de Proyectos Arquitectónicos, entre 1986-1992 y 1999-2010. Doctor Honoris Causa. Universidade Lusíada. Lisboa, junio 2005. Miembro titular de la Real Academia de Bellas Artes de Sant Jordi. Ver publicaciones en la web: www.arquitectonics.com

Correo electrónico de contacto: jose.muntanola@upc.edu

Dirección para la correspondencia: Escola Tècnica Superior d'Arquitectura de Barcelona. Avda. Diagonal 649. Planta 5a , puerta $2^{a} 08028$ Barcelona.

\section{Sergi Méndez Rodríguez}

Arquitecto. Personal docente investigador en formación, en el Grupo GIRAS. Arquitecto por la Escola Tècnica Superior d'Arquitectura del Vallès (2011). Máster en Teoría y Práctica del Proyecto de Arquitectura (2012). Estudiante de doctorado del Departamento de Proyectos de la Escuela Técnica Superior de Arquitectura de Barcelona, becado por la Generalitat de Catalunya (2013-2016). Colaboración en docencia en el Aula de Proyecto de Fin de Carrera (2015) y en el MUTTPA (2013). Correo electrónico de contacto: sergimendezrodriguez@gmail.com

\section{Júlia Beltrán Borràs}

Arquitecta. Personal docente investigador en formación, en el Grupo GIRAS. Arquitecta por la ETSAB (2011). Máster en Teoría y Práctica del Proyecto de Arquitectura (2013). Estudiante de doctorado del Departamento de Proyectos de la Escuela Técnica Superior de Arquitectura de Barcelona. Autora de varios artículos sobre la visión histórica y la dimensión social del espacio.

Correo electrónico de contacto: julsbel@gmail.com 